\title{
Chiral Motifs in Interpenetrated Metal-Organic Frameworks Formed from Achiral Tetrahedral Ligands
}

\author{
Qiang Wen, ${ }^{[a]}$ Maria Chiara di Gregorio, ${ }^{[a]}$ Linda J. W. Shimon, ${ }^{[b]}$ Eugeny V. Alexandrov, ${ }^{[c]}$ \\ Davide M. Proserpio, ${ }^{*[\mathrm{~d}]}$ Michal Lahav, ${ }^{\left[{ }^{[a]}\right.}$ and Milko E. van der Boom ${ }^{*[a]}$
}

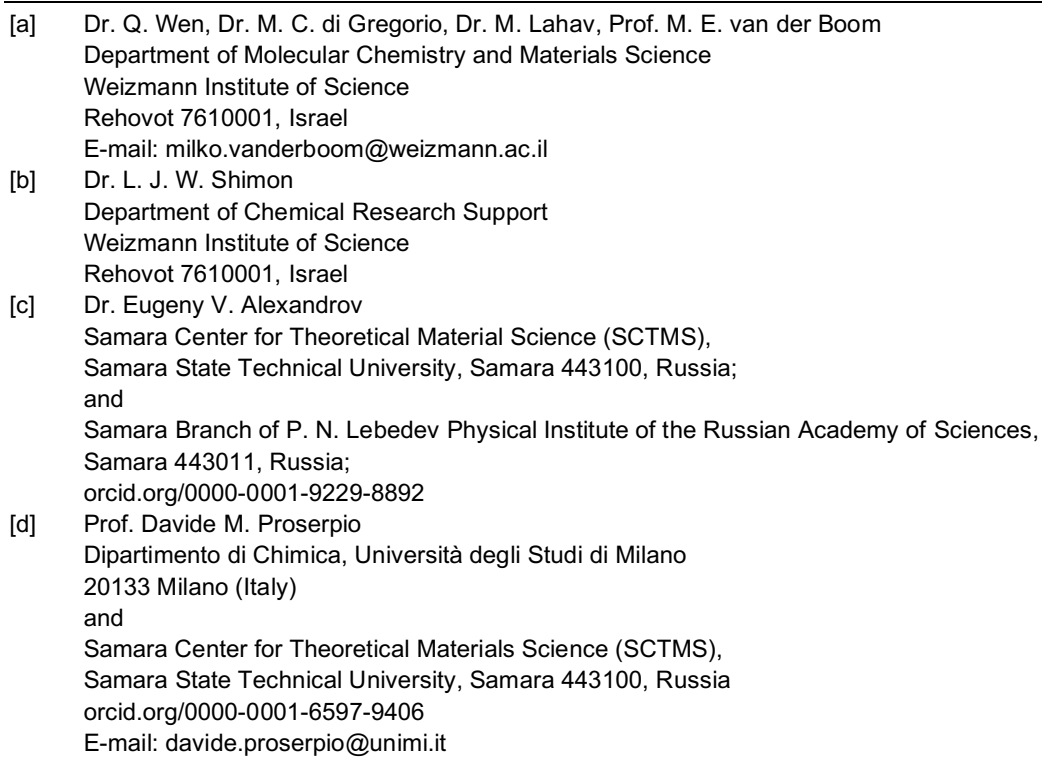

Supporting information for this article is given via a link at the end of the document.

\begin{abstract}
We demonstrate the formation of highly interpenetrated frameworks. An interesting observation is the presence of very large adamantane-shaped cages in a single network, making these crystals new entries in the collection of diamondoid-type metalorganic frameworks (MOFs). The frameworks were constructed by assembling tetrahedral pyridine ligands and copper dichloride. Currently, the networks' degree of interpenetration is among the highest reported and increases when the size of the ligand is increased. Usually, highly interpenetrated frameworks have low surface contact areas. In contrast, in our systems, the voids take up to $63 \%$ of the unit cell volume. The frameworks are chiral and formed from achiral components. The chirality is manifested by the coordination chemistry frameworks around the metal center, the structure of the helicoidal channels and the motifs of the individual networks. Channels with both chiralities are present within the unit cells. This phenomenon shapes the walls of the channels, which are composed of 10,16 , or 32 chains correlated to the degree of interpenetration 10-, 16- and 32-fold. By changing the distance between the center of the ligand and the coordination moieties, we succeeded to tune the diameter of the channels. Relatively large channels were formed, having diameters up to $31.0 \AA \times 14.8 \AA$.
\end{abstract}

Chirality is a key element of life and a desirable property in many functional materials. Better understanding of how it evolves and recognizing when it is present are important both for inferring general rules of fundamental interest and for enlarging the library of potential useful materials. ${ }^{[1-5]}$ Among the synthetic systems, metal-organic frameworks (MOFs) are crystalline coordination polymers formed by metallic nodes and organic ligands as connectors among the nodes. ${ }^{[6]}$ The metal-ligand interaction leads to molecular packing which may be porous and where both continuous channels and 3D cages can be present. In this class of material the chirality is mainly analyzed at the interior surface of pores and channels due to the possibility to exploit these areas for enantiomeric separation and chiral catalysis. $^{[7-9]}$ When enantiopure chiral ligands are used, MOFs are packed into Sohncke or chiral space groups. ${ }^{[10-12]}$ Such crystals often exhibit homochiral channels and are homochiral bulk solids. Interestingly, chiral MOFs can also be formed from achiral components. For example, Morris et al. demonstrated how solvents can induce homochirality in MOFs. ${ }^{[13]}$ Others have observed that racemic mixtures, chiral additives, and even impurities can yield chiral crystals. ${ }^{[14,15]}$ Such studies are relevant models for better understanding symmetry breaking in nature and in studies aimed at revealing the origin of life.$^{[16,17]}$ In spite of the large number of studies, designing chiral MOFs from achiral compounds without using additives remains a challenge. The possibility that one could also use achiral ligands to design chiral MOF's without the use of additives increases the pool of available ligands. Likewise, it is also difficult to predict a combination of structural properties such as porosity, network interpenetration, stability, morphology, and surface properties. ${ }^{[18,19]}$ For example, diamondoid-type structures have been demonstrated by coordination chemistry; they can display a rich isomerism of interpenetrating networks. ${ }^{[20,21]}$ The degree of interpenetration is related to the degree of porosity, which in turn, controls functionalities including guest absorption, catalysis, and sensing. ${ }^{[22,23]}$ Yang, Batten, $\mathrm{Ma}$, and co-workers reported an extraordinary interpenetrated MOF consisting of a 54-fold interpenetrated coordination framework. ${ }^{[24]}$

We previously reported that tetrahedral pyridine-based ligands generate metallo-organic single crystals having uniform and/or unusual multidomain morphology. ${ }^{[25,26]}$ Their growth, 
starting from these achiral ligands and metal salts, involves solvothermal processes and results in the formation of chiral molecular packing. The crystals are isostructural, regardless of the nickel or copper salts used and exhibit two different types of homochiral channels. The entire framework is completely held by the coordination of four pyridine groups in a propeller-like arrangement around the metal cations. The relationship between the achiral tetrahedral pyridine ligands, chiral molecular networks, and channel properties is complex.
In this study the coordination chemistry of three different but structurally related ligands with copper dichloride is reported. The structures we obtained confirm the tendency of this kind of ligand to generate chiral channels. ${ }^{[25-27]}$ These channels are formed by an unprecedented number of individual networks; the overall structures are among the highest reported interpenetrating frameworks. The combination of highly interpenetrated networks and continuous
A

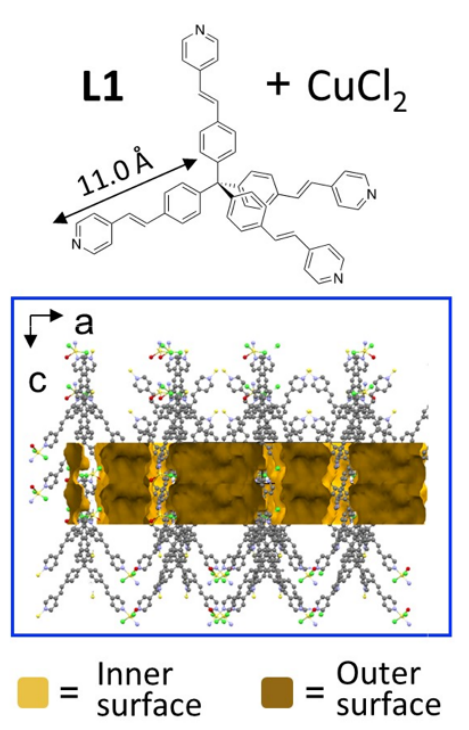

10-DIA

$$
\begin{aligned}
& =\mathrm{C} \\
& =\mathrm{O} \\
& =\mathrm{Cu} \\
& =\mathrm{N} \\
& =\mathrm{Cl}
\end{aligned}
$$$$
\downarrow \mathrm{a}
$$$$
\text { b }
$$

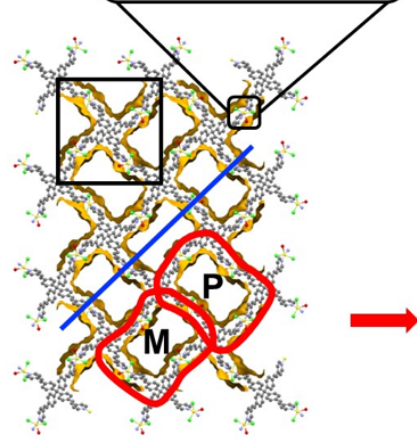

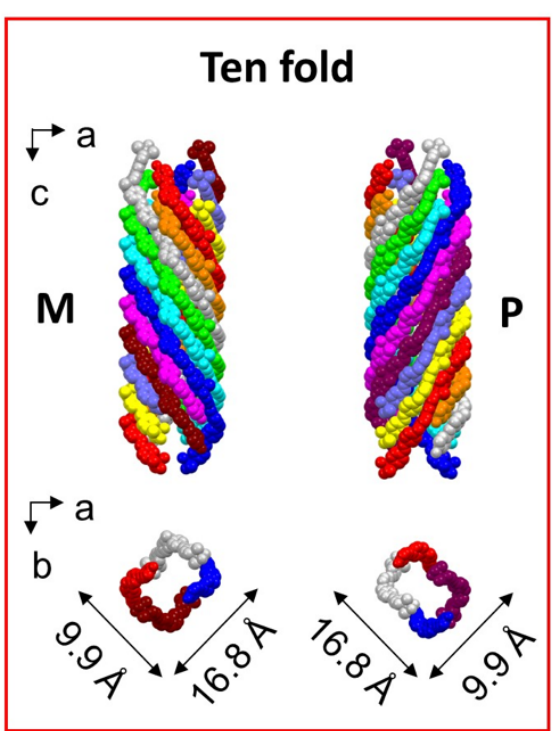

B

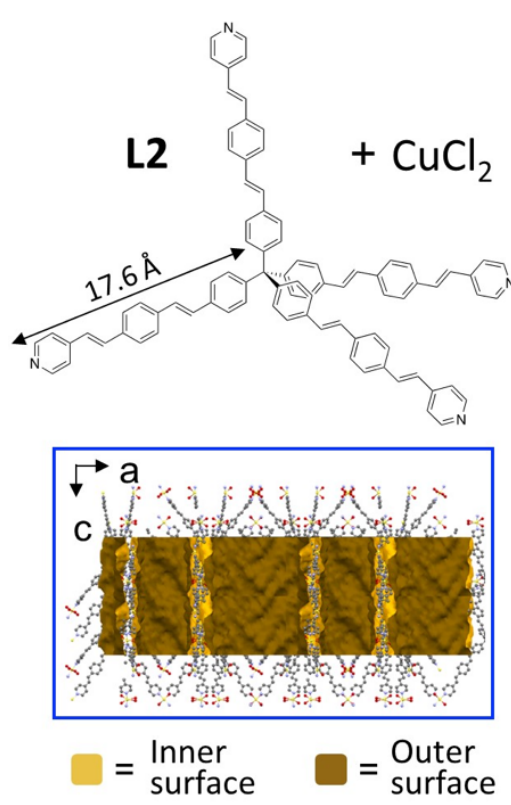

\section{6-DIA}

$$
\begin{aligned}
& =C \\
& =O \\
& =C U \\
& =N
\end{aligned}
$$

\section{$\checkmark a$}

b

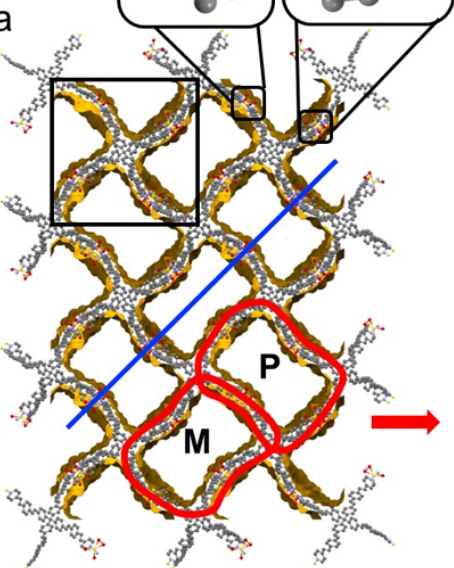

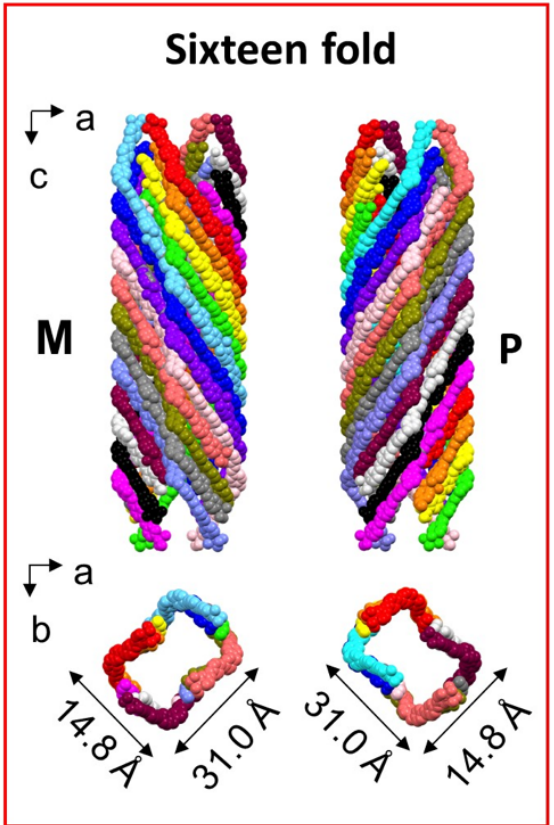

Figure 1. Single crystal X-ray structures of 10-DIA (A) and 16-DIA (B). Building blocks are reported in the top left. Connoly surface representations of the crystal structures down the $b$ (left, blue frames) and $c$ (center) axes. The channels are highlighted by red lines and their helicoidal packing is reported in the red frames (right). Zoom-in views of the coordination centers are reported in the black frames (top center). 

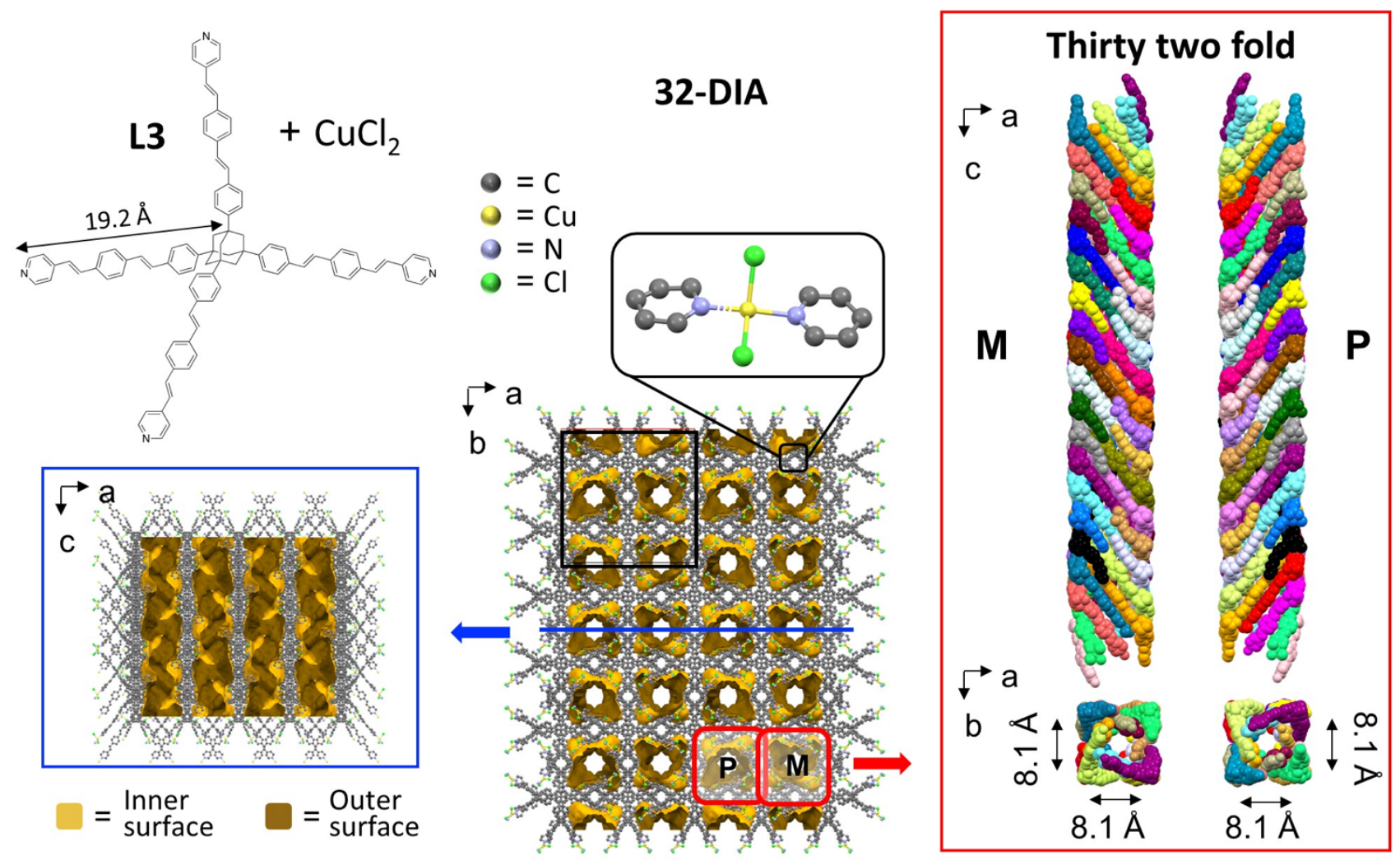

Figure 2. Single crystal X-ray structure of 32-DIA. Building blocks are reported in the top left. Connoly surface representations of the crystal structures down the $b$ (left, blue frames) and c (center) axes. The channels are highlighted by red lines and their helicoidal packing is reported in the red frames (right). Zoom-in views of the coordination center is reported in the black frames (top center

channels is not common; it results in high contact surface areas. Another fascinating structural aspect is the presence of huge adamantane cages.

The ligands (L1-L3) that we used are achiral, and owing to their tetrahedral symmetry $\left(T_{\mathrm{d}}\right)$, they are structurally fit for the general strategy for forming diamondoid framework structures. ${ }^{[28]}$ Robson and co-workers used this reticular approach to construct diamondoid structures using coordination of cyano groups with metal centers in 1989. ${ }^{[29]}$ Structurally, our ligands have four pyridine moieties and a carbon (L1 and L2) or an adamantane (L3) core (Figure 1 and 2, top left). The distance between the pyridine moieties of consecutive "arms", and the distance between the pyridine moieties and the core can be varied by using vinyl-phenyl groups. Yaghi and Schröder showed that elongation of the "arms" both in branched and linear ligands is a strategy to increase the dimensions of channels in MOFs. ${ }^{[30,31]}$ The coordinative chemistry of pyridine and divalent cations such as $\mathrm{Cu}^{2+}$ has been well established, and it is used for constructing sophisticated assemblies, as demonstrated by Sauvage ${ }^{[32]}$ Fujita ${ }^{[33]}$, and others. ${ }^{[34-37]}$ The three MOFs reported here were grown using copper dichloride, L1-L3, under solvothermal reaction conditions (DMF, $\mathrm{T}=105^{\circ} \mathrm{C}$ ). The vessels were gradually cooled down after $24 \mathrm{~h}$ of heating. In order to solubilize the ligands in DMF, we used small amounts of $\mathrm{HCl}$, which, by protonating the pyridine groups, enhances their polarity. This method may seem counterintuitive, as the coordination sites are actually inactivated; however, the formation of coordination-based crystals is thermodynamically favored. Single-crystal X-ray analysis revealed that 10-DIA and 16-DIA crystallize in non-centrosymmetric space groups $P-4$ and $P-4 n 2$, whereas 32-DIA crystallizes in a centrosymmetric space group, $14_{1} / \mathrm{acd}$. For these three MOFs, the framework structures consist of highly interpenetrated diamondoid-based networks (Figure S1 and S2). These networks are formed by coordinating two pyridine moieties belonging to two different ligands with $\mathrm{Cu}(\mathrm{II})^{[38]}$ (Figure 1 and 2, top center). In despite of the different anions and co-ligands $\left(\mathrm{Cl}^{-}, \mathrm{H}_{2} \mathrm{O}, \mathrm{O}_{2}\right)$, the structures of the constituting networks are characterized by adamantane-type cage motifs (Figure 3 ).

Two channels are present by a helical arrangement of the conjugated vinyl-pyridine moieties. The walls of the channels have an opposite chirality $(P$ and $M)$, and run along the $c$-axis (Figure 1 and 2, right). The crystals grown from L1 and L2 have a very similar structural appearance (e.g., the shape and disposition of the channels) in comparison with 32-DIA. For 10DIA and 16-DIA, the channels run along the 4-fold roto-inversion axis and for 32-DIA the channels are aligned 


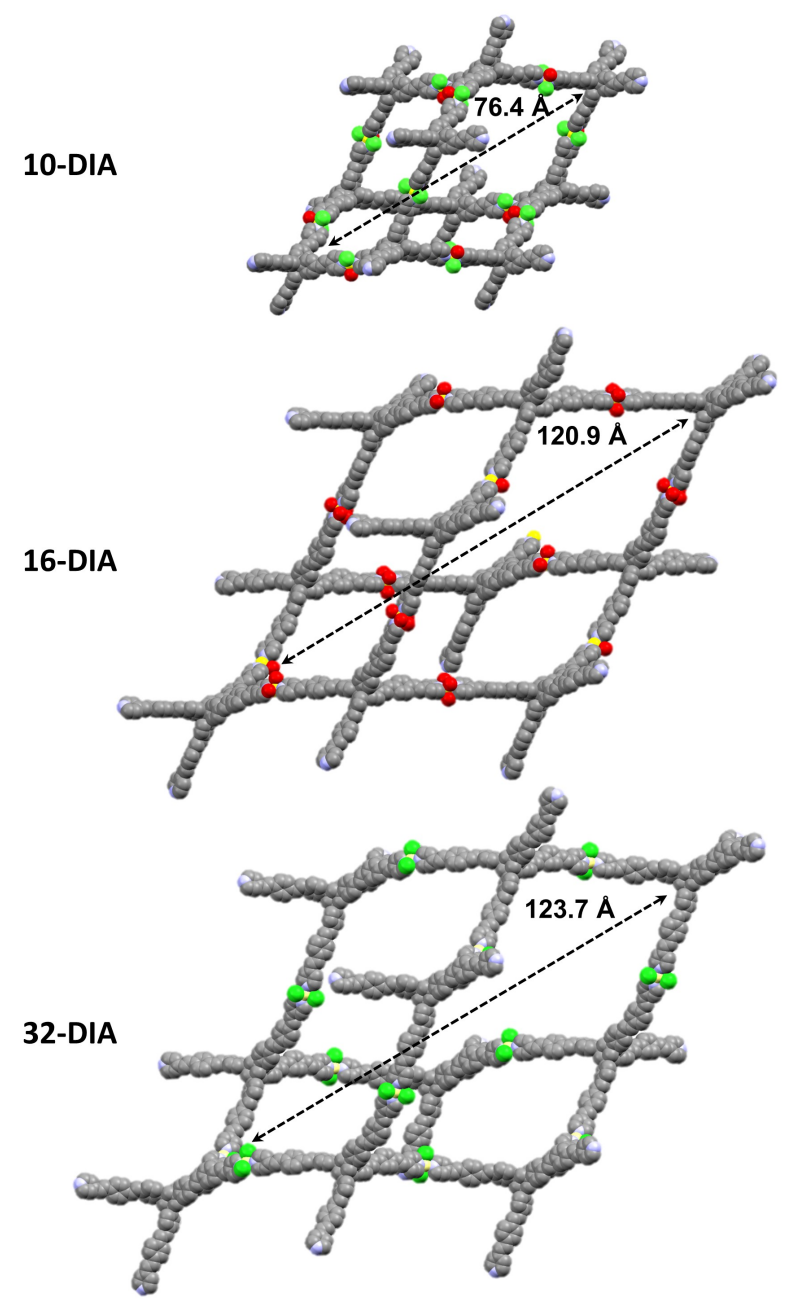

Figure 3. Space-filling representation of the adamantane-type cage motifs characterizing the constituting networks of 10-DIA (top), 16-DIA (center) and 32-DIA (bottom). Color code is yellow for copper, red for oxygen, green for chlorine, violet for nitrogen and grey for carbon.

along the $4_{1}$ screw axis. The channels of 10-DIA and 16-DIA are rectangular, having diameters of $16.8 \times 9.5 \AA$ and $31.0 \times 14.8 \AA$, respectively, whereas the channels of 32-DIA are square when viewed down the c-axis $(8.1 \times 8.1 \AA)$. Analysis by ToposPro ${ }^{[39]}$ shows that the number of networks, i.e. the degree of interpenetration, is 10, 16, and 32 for 10-DIA, 16-DIA, and 32DIA, respectively (Figure S4).

The interpenetration modes have been described in 2005 with a geometrical approach according to three classes ${ }^{[40]}$ and in 2012 with the topological types computing the Hopf ring nets, an approach that is independent of the geometrical embedding: different classes may be observed for the same topological type. ${ }^{[41]}$ Diamondoid nets are among the most frequently observed underlying nets ${ }^{[2,43]}$ and their modes of interpenetration are enumerated for the maximum symmetry embedding, i.e. with exact tetrahedral nodes, straight edges and transitivity [11] (one kind of node and one kind of edge). ${ }^{[44,45]}$ The comparison with the observed structures indeed shows that the smallest transitivity (number $[p q]$ of inequivalent nodes $p$ and edges $q$ ) is the one actually observed (Table S2).

10-DIA is 10 -fold interpenetrated of the class la, i.e. the ten nets are related by a single translation [001] that makes the ten nets eclipsed if view down the $c$ axis. 16-DIA is 16 -fold of class of IIla, where the translation vector [001] relates 8 interpenetrating networks, and the 8 other networks are related to former ones by two diagonal glide planes $n$ oriented as (100) and (010). What is peculiar here is that this geometry does not affects the topological type of entanglement that is the same as the one observed from 10-DIA, with all the nets related by a single translational vector. We can say that the two structures both present the most common interpenetration mode that aligns all nets equally spaced along the $\mathrm{S}_{4}$ axis of the adamantane cage (the so called "normal mode" described since $1998^{[46-48]}$ ).

32-DIA is 32-fold interpenetrated of the rarely seen class IIlc (Figures S2, S4 and S5), related by four translations and three non-translational symmetry elements $i, \mathrm{C}_{2}$, glide $d$. Interestingly, all the networks participate in the structure of the channel walls, akin to the strands of a rope. Complete details on the classes are reported in the supporting material (Figure S3).

A further analysis reveals that the three MOFs are build up by structurally similar individual networks having different dimensions. Viewed from the $S_{4}$ axis, there is a distortion of the adamantane-type cages that increase when moving from 10-DIA to 32-DIA. Interestingly, these networks are made by perpendicularly positioned helicoids having opposite handiness (Figures 4, S6 and S7). The overall crystal structures of the MOFs appear different because the interpenetration is masking the structures of the individual networks, especially for 32-DIA.

The largest dimension of the adamantane type cages also increases from $76.4 \AA$ (10-DIA) to $120.9 \AA$ (16-DIA); however, the cage dimensions for 16-DIA and 32-DIA are nearly identical (120.9 $\AA$ vs $123.7 \AA$ ). The number of interpenetrating networks for 32-DIA is remarkably high, indicating that the variation of the ligand core consistently affects the MOF packing. From 10-DIA to $16-\mathrm{DIA}$, the calculated porosity increases from $50.9 \%$ to $64.1 \%$ of the unit cell volume. As expected, the porosity decreases in 32-DIA (28.6\%) due the different mode of interpenetration. 
It is important to note that all three modes observed are of transitivity [11] (the topologically simplest structure is the one obtained) as the 15 of the 16 known examples of diamondoid nets with interpenetration 10 -fold or more as reported in the Table S2. Five 10-fold structures with CSD refcodes FUXCOS, PUQDOW, XISXAY, MOXPUL and one 12-fold OMUNER09 have similar 1-periodic helical channels of rectangular crosssection as observed in 10-DIA and 16-DIA because of similar "normal mode" of interpenetration. From the analysis of the three structures, we see the record 32-fold interpenetration of dia nets in 32-DIA arise from the longer ligand bridged with metal cations (L1: $11.0 \AA$, L2: $17.6 \AA$, L3: $19.2 \AA$ ). . Bridging metal cations elongating the edges also gives rise to other structures with the highest degrees of interpenetration 20 (KUVMEV) and 25 (ZEFHEZ). Some loose correlation could be seen relating the edge length and the angles at the mid edge as shown in the plots for the 16 structures (Figure S8). The size of accessible channels is also not always proportional to the elongation of linkers. Some of the straight edges of underlying net of 32-DIA are intersecting due to abnormal mutual orientation of nets, and so the walls of the channels are constructed by bent linkers (angle $147^{\circ}$ ) (Figure S4). In this way, the observed mode of interpenetration (abnormal) reduces pore dimensions more than the normal mode would and increases the density of the structure due to the larger number of nets filling the same space. The abnormal mode of interpenetration also prevents the presence of accessible channels in other six highly interpenetrating structures (FOKZEM, VOTQAY, WODYIZ, NUCJEB, ZEFHEZ, KUVMEV). How do the crystal structures for the MOFs comprising L1, L2, and L3 compare? The obvious differences lie in the core and in the "arm" dimensions. Looking at the ligands in the crystal packing, one can observe the following effects. All the ligands have similar distortions. Starting from symmetric pyramids (equilateral triangles as facets), where the ligands are inscribed with the pyridine units at the vertexes, deviations occur in asymmetric pyramids with isosceles triangles as facets (Figure S9). The ratio between the sides of the triangles is 1.3-1.5. The triangle angles are about $43^{\circ}$ and $68^{\circ}$. The volumes inscribing L1, L2 and L3 are 560.4, 2152.5 and $2895.1 \AA^{3}$ respectively. The "arms" of L3 are more curved. We noted that for both L2 and L3 the pyridine groups are rotated oppositely or coherently with respect to the phenyl rings. This results in two sets of two structurally different "arms" within one molecule. However, this effect is not observed in L1. Addressing the posed question is not trivial. The increase in the adamantane cage size, interpenetration level, and ligand curvature (bending) appears to be correlated. The increased size of the adamantane cages allows for a higher interpenetration of the networks. This structural effect is consistent with a higher bending of the ligand arms.

In conclusion, we showed that the poorly soluble ligands can be solubilized under acidic conditions and that they are still sufficiently reactive to coordinate to the copper centers. It is remarkable that protonation of the pyridine moieties eventually results in the formation of distorted diamondoid metal-organic frameworks. The chirality is expressed at different levels in these crystals since the constituting networks are themselves helicoids. Crystals having opposite chirality within the same unit cell were formed. Previously we have produced crystals with two types of homochiral channels. Changing the ligand core (carbon vs
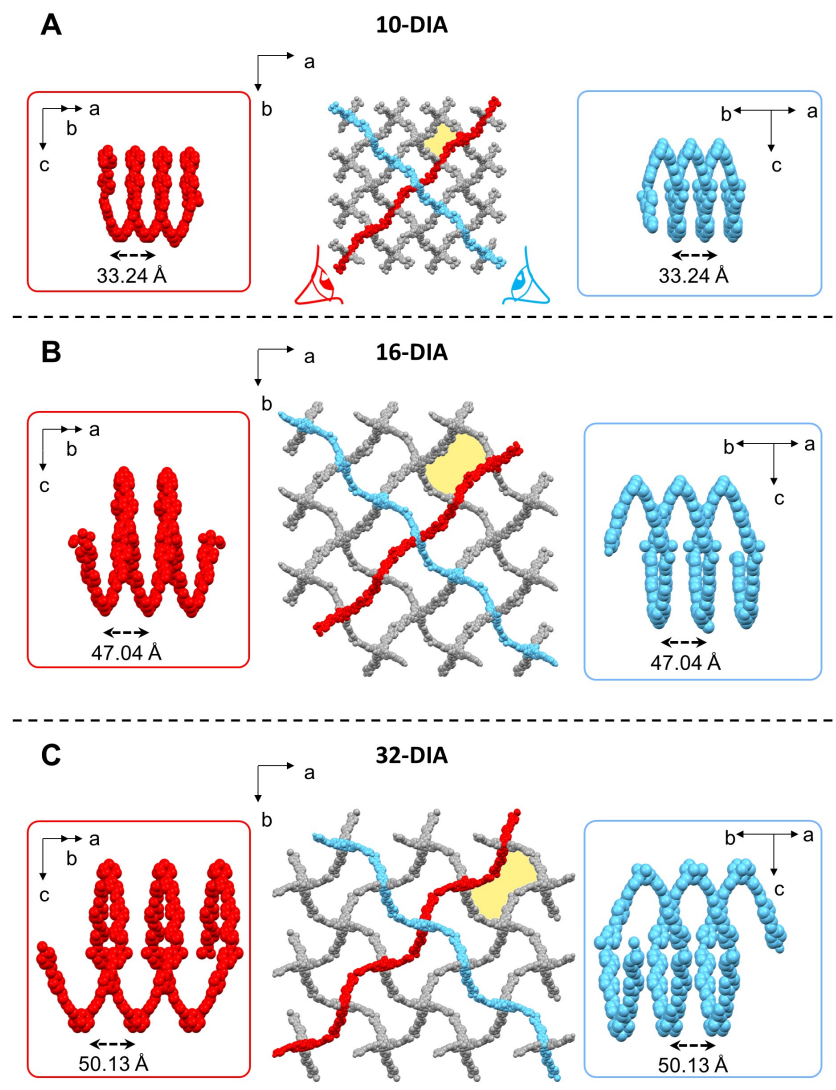

Figure 4. Structure of one network constituting 10-DIA (A), 16-DIA (B) and 32DIA (C). Helicoids of opposite handiness characterize these networks (red and cyan frames).

adamantane) drastically changes the overall appearance of the framework, including the shape of the channels. Changing only the length of the "arm" while maintaining the $C$ core affects the diameter of the channels while preserving the shape. It is interesting to note that the individual networks for the three MOFs are similar in shape but the level and mode of interpenetration is different. A high level of interpenetration usually goes hand in hand with a low solvent accessible surface and contact surface areas; however, the opposite was observed in our MOFs. In spite of the increasing level of interpenetration, the crystals still contain continuous channels, even in 32-DIA, with 32 interpenetrating networks and $28 \%$ porosity. The presence of the channels explains the relatively high porosity of these crystals. For example, $25^{[21]}$ and $54-$ fold $^{[24]}$ interpenetrated MOFs have a lower calculated solvent accessible surface and contact surface areas. A 32-fold interpenetrated hydrogen bond $^{[49]}$ assembly has a comparable porosity (Table S3).

The achiral tetrahedral ligands used in this and other related studies appear to have a general tendency to form assemblies with chiral channels, regardless of the crystallization conditions (acidic vs neutral conditions, $\mathrm{Cu}^{2+} \mathrm{vs}^{2+}$, counter ions) and ligand structure (core, bond order, and "arm" length). ${ }^{[25-27]}$ The channels' diameter, shape, and the nature of their chirality (homochiral vs heterochiral) appear to be a function of the ligand structure. Such structures with different channel topologies, offer opportunities for selective inclusion of different guest molecules. ${ }^{[50]}$ Moreover, the perpendicularly positioned chiral motifs having opposite handiness indicates that 
such crystals can have interesting directional chiroptical properties. ${ }^{[51,52]}$

\section{Acknowledgements}

This research was supported by the Israel Science Foundation (ISF), the Minerva Foundation, and the Helen and Martin Kimmel Center for Molecular Design. M.E.v.d.B. holds the Bruce A. Pearlman Professional Chair in Synthetic Organic Chemistry. D.M.P. thanks Igor A. Baburin for discussion. E.V.A. is grateful to the Russian Science Foundation for supporting topological methods of crystal structures analysis (Grant No. 18-73-10116).

Keywords: metal-organic frameworks • interpenetration • channels $\bullet$ symmetry breaking $\bullet$ diamondoid

\section{References}

[1] H. Kuang, C. Xu, Z. Tang, Adv. Mater. 2020, 32, 1-3.

[2] C. Viedma, Phys. Rev. Lett. 2005, 94, 3-6.

[3] A. G. Shtukenberg, Y. O. Punin, A. Gujral, B. Kahr, Angew. Chem. Int. Ed. 2014, 53, 672-699.

[4] A. Casnati, R. Liantonio, P. Metrangolo, G. Resnati, R. Ungaro, F. Ugozzoli, Angew. Chem. Int. Ed. 2006, 45, 1915-1918.

[5] W. Meng, J. K. Clegg, J. D. Thoburn, J. R. Nitschke, J. Am. Chem. Soc. 2011, 133, 13652-13660.

[6] H. Furukawa, K. E. Cordova, M. O’Keeffe, O. M. Yaghi, Science 2010 9, 1230444.

[7] L. Ma, C. Abney, W. Lin, Chem. Soc. Rev. 2009, 38, 1248-1256.

[8] D. N. Dybtsev, K. P. Bryliakov, Coord. Chem. Rev. 2021, 437, 213845.

[9] J. Seo, D. Whang, H. Lee, S. Jun, J. Oh, Y. Jeon, K. Kim, Nature 2000, 404, 982-986.

[10] P. Larpent, A. Jouaiti, N. Kyritsakas, M. W. Hosseini, CrystEngComm 2019, 21, 2534-2540.

[11] D. Bradshaw, J. B. Claridge, E. J. Cussen, T. J. Prior, M. J. Rosseinsky, Acc. Chem. Res. 2005, 38, 273-282.

[12] Y. Pre, P. Nitrides, E. P. K. Farbko, Nature 2000, 404, 982-986.

[13] Z. Lin, A. M. Z. Slawin, R. E. Morris, J. Am. Chem. Soc. 2007, 129, 4880-4881.

[14] R. E. Morris, X. Bu, Nat. Chem. 2010, 2, 353-361.

[15] D. Wu, K. Zhou, J. Tian, C. Liu, J. Tian, F. Jiang, D. Yuan, J. Zhang, Q. Chen, M. Hong, Angew. Chem. Int. Ed. 2021, 60, 3087-3094.

[16] G. H. Wagnière, On Chirality and the Universal Asymmetry: Reflections on Image and Mirror Image, 2007.

[17] I. Weissbuch, G. Bolbach, L. Leiserowitz, M. Lahav, Science 2004, 34, 79-92.

[18] O. Shekhah, H. Wang, M. Paradinas, C. Ocal, B. Schupbach, A. Terfort, D. Zacher, R. A. Fischer, C. Woll, Nat Mater 2009, 8, 481-484.

[19] X. Zhang, Z. Chen, X. Liu, S. L. Hanna, X. Wang, R. Taheri-Ledari, A. Maleki, P. Li, O. K. Farha, Chem. Soc. Rev. 2020, 49, 7406-7427.

[20] L. Cao, P. Wang, X. Miao, Y. Dong, H. Wang, H. Duan, Y. Yu, X. Li, P. J. Stang, J. Am. Chem. Soc. 2018, 140, 7005-7011.

[21] Y. P. He, Y. X. Tan, J. Zhang, CrystEngComm 2012, 14, 6359-6361.

[22] H. L. Jiang, T. A. Makal, H. C. Zhou, Coord. Chem. Rev. 2013, 257 2232-2249.

[23] M. Gupta, J. J. Vittal, Coord. Chem. Rev. 2021, 435, 213789

[24] H. Wu, J. Yang, Z. M. Su, S. R. Batten, J. F. Ma, J. Am. Chem. Soc. 2011, 133, 11406-11409.

[25] M. C. di Gregorio, L. J. W. Shimon, V. Brumfeld, L. Houben, M. Lahav, M. E. van der Boom, Nat. Commun. 2020, 11, 380.

[26] M. C. di Gregorio, M. Elsousou, Q. Wen, L. J. W. Shimon, V. Brumfeld, L. Houben, M. Lahav, M. E. van der Boom, Nat. Commun. 2021, 12, 957.
[27] Q. Wen, S. Tenenholtz, L. J. W. Shimon, O. Bar-Elli, L. M. Beck, L. Houben, S. R. Cohen, Y. Feldman, D. Oron, M. Lahav, M. E. Van Der Boom, J. Am. Chem. Soc. 2020, 142, 14210-14221.

[28] M. J. Zaworotko, Chem. Soc. Rev. 1994, 23, 283-288.

[29] B. F. Hoskins, R. Robson, J. Am. Chem. Soc., 1989, 111, 5962-5964

[30] H. Deng, S. Grunder, K. E. Cordova, C. Valente, H. Furukawa, M. Hmadeh, F. Gándara, A. C. Whalley, Z. Liu, S. Asahina, H. Kazumori, M. O'Keeffe, O. Terasaki, J. F. Stoddart, O. M. Yaghi, Science 2012, 336, 1018-1023.

[31] F. Moreau, D. I. Kolokolov, A. G. Stepanov, T. L. Easun, A. Dailly, W. Lewis, A. J. Blake, H. Nowell, M. J. Lennox, E. Besley, S. Yang, M. Schröder, Proc. Natl. Acad. Sci. U. S. A. 2017, 114, 6-11.

[32] J. P. Sauvage, Acc. Chem. Res. 1998, 31, 611-619.

[33] M. Yoshizawa, K. Ono, K. Kumazawa, T. Kato, M. Fujita, J. Am. Chem. Soc. 2005, 127, 10800-10801.

[34] S. S. Kaye, J. R. Long, J. Am. Chem. Soc. 2005, 127, 6506-6507.

[35] J. H. Dou, L. Sun, Y. Ge, W. Li, C. H. Hendon, J. Li, S. Gul, J. Yano, E. A. Stach, M. Dincă, J. Am. Chem. Soc. 2017, 139, 13608-13611.

[36] M. C. di Gregorio, P. Ranjan, L. Houben, L. J. W. Shimon, K. Rechav, M. Lahav, V, M. E. an der Boom, J. Am. Chem. Soc. 2018, 140, 91329139

[37] A. H. M. De Vries, A. Meetsma, B. L. Feringa, Angew. Chemie 1996, 35, 2374-2376

[38] R. J. Dubois, C. P. Landee, M. Rademeyer, M. M. Turnbull, J. Coord Chem. 2019, 72, 1785-1809.

[39] V. A. Blatov, A. P. Shevchenko, D. M. Proserpio, Cryst. Growth Des 2014, 14, 3576-3586.

[40] I. A. Baburin, V. A. Blatov, L. Carlucci, G. Ciani, D. M. Proserpio, J. Solid State Chem. 2005, 178, 2452-2474

[41] E. V. Alexandrov, V. A. Blatov, D. M. Proserpio, Acta Crystallogr. Sect. A: Found. Crystallogr. 2012, 68, 484-493.

[42] E. V. Alexandrov, V. A. Blatov, A. V. Kochetkov, D. M. Proserpio, CrystEngComm 2011, 13, 3947-3958.

[43] A. P. Shevchenko, E. V. Alexandrov, A. A. Golov, O. A. Blatova, A. S Duyunova, V. A. Blatov, Chemical Communications 2020, 56, 96169619

[44] C. Bonneau, M. O'Keeffe, Acta Crystallogr. Sect. A: Found. Crystallogr. 2015, 71, 82-91.

[45] I. A. Baburin, Acta Crystallogr. Sect. A: Found. Crystallogr. 2016, 72, 366-375.

[46] S. R. Batten, R. Robson, Angew. Chem. Int. Ed. 1998, 37, 1460-1494.

[47] S. R. Batten, CrystEngComm 2001, 3, 67-72.

[48] L. Carlucci, G. Ciani, D. M. Proserpio, S. Rizzato, Chem.-Eur. J. 2002, 8, 1519-1526

[49] S. A. Boer, P. X. Wang, M. J. MacLachlan, N. G. White, Cryst. Growth Des. 2019, 19, 4829-4835.

[50] J. R. Li, R. J. Kuppler, H. C. Zhou, Chem. Soc. Rev. 2009, 38, $1477-$ 1504

[51] L.-L. Xu, H.-F. Zhang, M. Li, S. W. Ng, J.-H. Feng, J.-G. Mao, D. Li J. Am. Chem. Soc. 2018, 140, 11569-11572.

[52] J. K. O'Loane Chem. Rev. 1980, 80, 1, 41-61. 


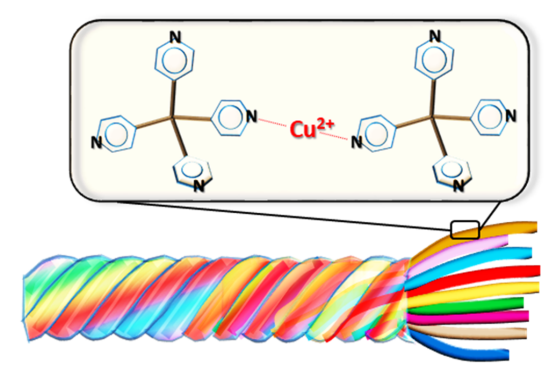

Tetragonal achiral ligands and copper ions assemble into a record number of interpenetrating diamondoid networks. The networks intertwine as strands of a molecular rope to shape chiral channels. The design of the ligand "arms" allows for the modulation of the interpenetration degree and channel properties thereof. The concurrent presence of such numerous interpenetrated networks and porosity is unprecedented for metal-organic frameworks. 